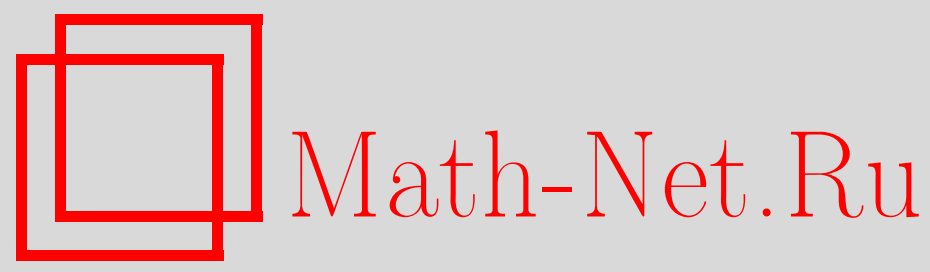

В. В. Филиппов, Об условии равностепенной непрерывности последовательности пространств решений, Матем. заметки, 1997, том 61, выпуск 3, 416-423

DOI: https://doi.org/10.4213/mzm1515

Использование Общероссийского математического портала Math-Net.Ru подразумевает, что вы прочитали и согласны с пользовательским соглашением http://www.mathnet.ru/rus/agreement

Параметры загрузки:

IP : 54.157 .27 .8

26 апреля 2023 г., $15: 42: 37$ 


\section{ОБ УСЛОВИИ РАВНОСТЕПЕННОЙ НЕПРЕРЫВНОСТИ ПОСЛЕДОВАТЕЛЬНОСТИ ПРОСТРАНСТВ РЕШЕНИЙ}

\section{В.В. Филиппов}

Проверкавыполнения должньп образом сформулированного условия равностепенной непрерывности последовательности пространств решений является одним из двух ключевых моментов теории задачи Коши для уравнений с особенностями правой части. В заметке получен соответствующий достаточный признак.

Библиограффия: 9 названий.

В [1]-[3] было начато развитие нового подхода к теории обыкновенных дифференциальных уравнений, направленного, в частности, на применение к исследованию уравнений с разрывной правой частью и соответствующих дифференциальных включений и основанного на систематическом использовании введенных там топологических структур. В [4]-[9] этот подход получил дальнейшее развитие. В центре исследования оказывается понятие сходимости последовательности пространств решений, адекватное идее о непрерывной зависимости решений от параметров уравнений, а вместе с ним - вопрос об указании достаточных условий такой сходимости. Общая схема доказательства такой сходимости обсуждена в [3], [4], [7]-[9]. Наша цель в этой заметке- получение утверждения, удобного для проверки условия равностепенной непрерывности последовательности пространств решений, что является одним из двух ключевых моментов при доказательстве сходимости. Мы получим результат, положение которого аналогично положению теоремы 4 из [3] и теоремы 1 из [4].

Частью обозначений из [1]-[9] будем пользоваться без дополнительных пояснений.

Пусть $U$ - открытое подмножество произведения $\mathbb{R} \times \mathbb{R}^{n}$. Выделим класс многозначных отображений $F: U \rightarrow \mathbb{R}^{n}$, удовлетворяющих условию

(1) значения отображения $F$ - непустые выпуклые (односторонние) конусы с вершиной в начале координат (при этом $\{\overline{0}\}$ допускается в качестве значения).

Приступая к основной части изложения, зафиксируем

(2) $F, F_{i}: U \rightarrow \mathbb{R}^{n}, i=1,2, \ldots,-$ многозначные отображения, каждое из которых удовлетворяет условию (1), причем

(3) для любой точки $x \in U$

$$
\begin{gathered}
F(x) \supseteq \cap\left\{\operatorname{cc}\left(\cup\left\{F_{i}(O x): i=k, k+1, \ldots\right\} \cup F(O x)\right): k=1,2, \ldots,\right. \\
O x \text { пробегает множество всех окрестностей точки } x \text { в } U\}
\end{gathered}
$$

(здесь сс $(M)$ обозначает замкнутую вьпуклую оболочку множества $M \subseteq \mathbb{R}^{n}$ ). 


\section{Пусть также}

(4) $\alpha_{i}: \mathbb{R} \rightarrow[0, \infty[, i=1,2, \ldots,-$ интегрируемые по Лебегу функции, их первообразные составляют равностепенно непрерывное семейство, $S_{i}(t)=\{u: u \in \mathbb{R}$, $\left.\|u\| \leqslant \alpha_{i}(t)\right\}$

(5) $G_{i}(t, y)=F_{i}(t, y)+S_{i}(t)=\left\{u+v: u \in F_{i}(t, y), v \in S_{i}(t)\right\}$ для $(t, y) \in U$, $i=1,2, \ldots$.

Для конуса $V$ с вершиной в начале координат и $\varepsilon>0$ обозначим через $P_{\varepsilon} \varepsilon$-окрестность множества $\{u: u \in V,\|u\|=1\}$ на единичной сфере $S$ и через $V_{\varepsilon}-$ конус

$$
\{\overline{0}\} \cup\left\{u: u \in \mathbb{R}^{n}, u \neq \overline{0}, \frac{u}{\|u\|} \in P_{\varepsilon}\right\} .
$$

Обозначим через $\tau(F, t)$ топологию на множестве $U_{t}=\left\{y: y \in \mathbb{R}^{n},(t, y) \in U\right\}$, базу которой в текущей точке $y \in U_{t}$ составляет семейство

$$
\left\{y \pm(F(t, y))_{\varepsilon} \cap O_{\delta} \overline{0}: \varepsilon, \delta>0\right\} .
$$

Обозначим через $C_{s}(U)$ множество всех определенных на всевозможных отрезках и одноточечных подмножествах действительной прямой $\mathbb{R}$ непрерывных функций со значениями в пространстве $\mathbb{R}^{n}$, графики которых лежат в множестве $U$.

Пусть $\pi(z)$ обозначает область определения, а $\operatorname{Gr}(z)$ - график функции $z$.

Мы рассматриваем $C_{s}(U)$ в качестве метрического пространства, считая расстоянием между двумя его элементами расстояние Хаусдорфа между их графиками (при этом расстояние Хаусдорфа является метризацией топологии Виеториса, и поэтому возникающая топология на самом деле не зависит от того, какая конкретно метрика рассматривается на множестве $U \subseteq \mathbb{R} \times \mathbb{R}^{n}$ (см. [1]-[3], [7]).

Пусть $G: U \rightarrow \mathbb{R}^{n}$ - многозначное отображение (соответственно $g: U \rightarrow \mathbb{R}^{n}$ - однозначное отображение). Следуя [1]-[3], функцию $z \in C_{s}(U)$ назьваем решением дифференциального включения $y^{\prime} \in G(t, y)$ (соответственно уравнения $y^{\prime}=g(t, y)$ ), если либо

а) функция $z$ обобщенно абсолютно непрерьвна и $z^{\prime}(t) \in G(t, z(t))$ (соответственно $z^{\prime}(t)=g(t, z(t))$, здесь и ниже речь идет об аппроксимативных (обобщенных) производньгх) почти при всех $t \in \pi(z)$, либо

б) область определения $\pi(z)$ функции $z$ состоит из одной точки.

Множество всех определенных таким образом решений включения $y^{\prime} \in G(t, y)$ (уравнения $\left.y^{\prime}=g(t, y)\right)$ обозначим через $D(G)(D(g))$. Будем назьвать его пространством решений включения $y^{\prime} \in G(t, y)$ (уравнения $\left.y^{\prime}=g(t, y)\right)$.

Для любого пространства $Z \subseteq C_{s}(U)$ и $M \subseteq U$ обозначим

$$
Z_{M}=\{z: z \in Z, \operatorname{Gr}(z) \subseteq M\} .
$$

Положим $D(G, M)=(D(G))_{M}$.

Наша цель - исследовать, ког да последовательность пространств

$$
Z_{i} \subseteq D\left(G_{i}\right), \quad i=1,2, \ldots,
$$

удовлетворяет условию

(s) для любого компакта $K \subseteq U$ любая последовательность функций $z_{j} \in\left(Z_{i_{j}}\right)_{K}$ $\left(i_{1}<i_{2}<\cdots\right)$ равностепенно непрерывна. 
Множество всех последовательностей $\left\{Z_{i}: i=1,2, \ldots\right\} \subseteq R^{i}(U)$, удовлетворяющих условию (s), обозначим через $s(U)$.

ТЕОРема. Пусть выполнены условия (1)-(6), $\gamma$-семейство подмножсеств множества $U$, для любого әлемента $V$ которого $\left\{\left(Z_{i}\right)_{V}: i=1,2, \ldots\right\} \in s(V)$, $E=U \backslash(\cup \gamma), u$ для любого $t \in \mathbb{R}$ множество $E_{t}$ не содержит (неодноточечных) континуумов, на которых топология $\tau(F, t)$ индуцирует ту же топологию, что и евклидова топология пространства $\mathbb{R}^{n}$. Тогда $\left\{\left(Z_{i}\right): i=1,2, \ldots\right\} \in s(U)$.

ДоказАтельство. І. Допустим противное. Тогда найдутся компакт $K \subseteq U$ и последовательность функций $z_{j} \in\left(Z_{i_{j}}\right)_{K}$, не являющаяся равностепенно непрерьвной, т.е. при некотором $\varepsilon>0$ и любом $m=1,2, \ldots$ бесконечно множество $A_{m} \subseteq \mathbb{N}$ индексов $k \in \mathbb{N}$, удовлетворяющих условию

(7) найдутся такие точки $s<t$ области определения $\pi\left(z_{m}\right)$ функции $z_{m}$, что $t-s<2^{-m}$ и $\left\|z_{k}(t)-z_{k}(s)\right\| \geqslant \varepsilon$.

Так как $A_{1} \supseteq A_{2} \supseteq A_{3} \supseteq \cdots$, то формула

$$
j_{1}=\min A_{1}, \quad j_{i}=\min \left(A_{i} \backslash\left\{j_{1}, \ldots, j_{i-1}\right\}\right) \quad \text { для } i=2,3, \ldots
$$

задает бесконечную последовательность индексов $A=\left\{j_{1}, j_{2}, \ldots\right\}$. Для $j_{m} \in A$ укажем пару точек $s_{j_{m}}<t_{j_{m}}$ из $\pi\left(z_{j_{m}}\right)$ в соответствии с условием (7):

$$
t_{j_{m}}-s_{j_{m}}<2^{-m} \text { и }\left\|z_{j_{m}}\left(t_{j_{m}}\right)-z_{j_{m}}\left(s_{j_{m}}\right)\right\| \geqslant \varepsilon .
$$

Таким образом,

$$
\begin{gathered}
t_{j}-s_{j} \rightarrow 0 \quad \text { при } j \in A \text { и } j \rightarrow \infty, \\
\left\|z_{j}\left(t_{j}\right)-z_{j}\left(s_{j}\right)\right\| \geqslant \varepsilon \quad \text { при } j \in A .
\end{gathered}
$$

Проекция $P$ компакта $K$ на первый сомножитель произведения $\mathbb{R} \times \mathbb{R}^{n}$ компактна, и поэтому из последовательности $\left\{s_{j}: j \in A\right\}$ мы можем выбрать подпоследовательность $\left\{s_{j}: j \in B\right\}$, сходящуюся к некоторой точке $s \in P$. Из условия (8) следует, что $s=\lim _{j \in B, j \rightarrow \infty} t_{j}$.

Пространство замкнутых подмножеств $\exp K$ компакта $K$ компактно (см., например, [8, теорема 4.5.1]), и поэтому из последовательности $\left\{z_{j}: j \in B\right\}$ мы можем выбрать подпоследовательность $\left\{z_{j}: j \in B_{1}\right\}$ таким образом, чтобы последовательность множеств $\left\{\operatorname{Gr}\left(z_{j}\right): j \in B_{1}\right\}$ имела в пространстве $\exp K$ некоторьй предел $H$. Так как проекция множества $\operatorname{Gr}\left(\left.z_{j}\right|_{\left[s_{j}, t_{j}\right]}\right)$ на первьй сомножитель произведения $\mathbb{R} \times \mathbb{R}^{n}$ совпадает с отрезком $\left[s_{j}, t_{j}\right]$, то в силу условия $s=\lim _{i \rightarrow \infty} s_{j}=\lim _{i \rightarrow \infty} t_{j}$ проекция компакта $H$ на первый сомножитель произведения $\mathbb{R} \times \mathbb{R}^{n}$ совпадает с точкой $s$. Из условия (9) следует, что диаметр компакта $H$ не меньше $\varepsilon$, поэтому он неодноточечен. Будучи пределом связных компактов, компакт $H$ связен, (см. [8, теорема 4.8.8]).

II. Покажем, что $H \subseteq U \backslash(\cup \gamma)$.

Допустим противное, т.е. существование точки $\left(t, y_{0}\right) \in H \backslash(\cup \gamma)$. При некотором $\eta>0$

(10) $H \backslash O_{\eta} y_{0} \neq \varnothing$,

(11) множество $\left[O_{\eta} y_{0}\right]$ лежит в некотором элементе $V$ семейства $\gamma$. 
В силу условия $\left(s, y_{0}\right) \in H$ мы можем так отметить точки $p_{j} \in\left[s_{j}, t_{j}\right]$, что $z_{j}\left(p_{j}\right) \rightarrow y_{0}$ при $j \in B_{1}$ и $j \rightarrow \infty$.

Из последней сходимости следует бесконечность множества

$$
B_{2}=\left\{j: j \in B_{1},\left(p_{j}, z_{j}\left(p_{j}\right)\right) \in O_{\eta}\left(s, y_{0}\right)\right\}
$$

Пусть для $j \in B_{2} I_{j}$ обозначает максимальный отрезок, лежащий в $\left[s_{j}, t_{j}\right]$ и удовлетворяющий условиям $p_{j} \in I_{j}, \operatorname{Gr}\left(\left.z_{j}\right|_{I_{j}}\right) \subseteq\left[O_{\eta}\left(s, y_{0}\right)\right]$.

Из условия (10) следует, что у последовательности множеств $\left\{\operatorname{Gr}\left(\left.z_{j}\right|_{I_{j}}\right): j \in B_{2}\right\}$ есть предельные точки вне $O_{\eta} y_{0}$, но отсюда вытекает, что последовательность функций $\left\{\left.z_{j}\right|_{I_{j}}: j \in B_{2}\right\}$ не является равностепенно непрерьвной.

Последнее противоречит условиям $(11)$ и $\left\{\left(Z_{i}\right)_{V}: i=1,2, \ldots\right\} \in s(V)$.

III. Предположим, что континуум $H$ лежит в плоскости $\lambda, \Lambda$ - пространство векторов плоскости $\lambda$. Если во всех точках множества $H$ или хотя бы его непустого открытого подмножества их указанные вьше окрестности в $U_{s}$ относительно топологии $\tau(F, s)$ содержат $\delta$-шары плоскости $\lambda$, то индуцированные топологии совпадают, и в этом случае теорема доказана.

Проанализируем случай, когда для некоторой точки $x_{1}=\left(t, y_{1}\right) \in H$ конус $F\left(t, y_{1}\right)$ не содержит полупространства пространства $\Lambda$.

Множество cс $\left(\cup\left\{F_{i}\left(O x_{1}\right): i=k, k+1, \ldots\right\} \cup F\left(O x_{1}\right)\right)$ в формуле, фигурирующей в условии (3), является замкнутым конусом, и поэтому у нас есть две возможности.

А. Начало координат $\overline{0}$ не принадлежит внутренности множества

$$
\operatorname{cc}\left(\cup\left\{F_{i}\left(O x_{1}\right): i=k, k+1, \ldots\right\} \cup F\left(O x_{1}\right)\right) .
$$

Б. Начало координат принадлежит внутренности множества

$$
\operatorname{cc}\left(\cup\left\{F_{i}\left(O x_{1}\right): i=k, k+1, \ldots\right\} \cup F\left(O x_{1}\right)\right)
$$

и тогда, как легко видеть,

$$
\operatorname{cc}\left(\cup\left\{F_{i}\left(O x_{1}\right): i=k, k+1, \ldots\right\} \cup F\left(O x_{1}\right)\right)=\Lambda \text {. }
$$

Если условие Б справедливо для любой окрестности $O x_{1}$ точки $x_{1}$ и любого $k=$ $1,2, \ldots$, то в силу $(3) F\left(x_{1}\right) \supseteq \Lambda$, но тогда база в точке $y_{1}$ топологии $\tau(F, s)$ совпадает с базой евклидовой топологии, что выходит за рамки рассматриваемого случая. Таким образом, для некоторой окрестности $O x_{1}$ точки $x_{1}$ вьполнено условие A.

При некотором $\mu>0[s-\mu, s+\mu] \times\left[O_{\mu} y_{1}\right] \subseteq O x_{1}$.

Пусть $\mathbf{e}_{0}$ - точка единичной сферы пространства $\Lambda$, наиболее удаленная от конуса

$$
\operatorname{cc}\left(\cup\left\{F_{i}\left([s-\mu, s+\mu] \times\left[O_{\mu} y_{1}\right]\right): i=k, k+1, \ldots\right\} \cup F\left([s-\mu, s+\mu] \times\left[O_{\mu} y_{1}\right]\right)\right) .
$$

Покажем, что $\left(\mathbf{e}_{0}, u\right) \leqslant 0$ для любого вектора

$$
u \in \operatorname{cc}\left(\cup\left\{F_{i}\left([s-\mu, s+\mu] \times\left[O_{\mu} y_{1}\right]\right): i=k, k+1, \ldots\right\} \cup F\left([s-\mu, s+\mu] \times\left[O_{\mu} y_{1}\right]\right)\right) .
$$


Допустив противное, получим, что условию (12) удовлетворяет вектор $u_{0}$, являющийся ближайшим к $\mathbf{e}_{0}$ элементом множества $(*)$. У нас есть две возможности.

1. Есть еще один (отличньй от $u_{0}$ ) вектор $u_{1}$, для которого $\left\|\mathbf{e}_{0}-u_{1}\right\|=\left\|\mathbf{e}_{0}-u_{0}\right\|$. Но тогда кратчайшая дуга, соединяющая точки $u_{0}$ и $u_{1}$ на сфере $S$ целиком лежит в конусе $(*)$ и любая ее неконцевая точка будет ближе к $\mathbf{e}_{0}$, чем $u_{0}$ и $u_{1}$, что противоречит указанию этих векторов.

2. Вектор $u_{0}$ является единственным ближайшим к $\mathbf{e}_{0}$ вектором из $(*)$. В этом случае можно удаляясь от $u_{0}$ по дуге большого круга, проходящего через $u_{0}$ и $u_{1}$, указать вектор $\mathbf{e}_{1}$, более удаленный от конуса $(*)$, чем вектор $\mathbf{e}_{0}$.

Таким образом, сделанное допущение неверно, и в силу доказанного для вектора $\mathbf{e}=-\mathbf{e}_{0}$ и указанного $\mu>0$

(13) $(\mathbf{e}, u) \geqslant 0$ для любого вектора (12).

IV. Вернемся к обозначениям I, сохраняя обозначения III. Пусть выполнено условие (13).

По [8, теорема 4.3.10] мы можем так отметить точки $r_{j} \in\left[s_{j}, t_{j}\right]$, что $z_{j}\left(r_{j}\right) \rightarrow y_{1}$ при $j \in B_{1}, j \rightarrow \infty$.

Множество $B_{2}=\left\{j: j \in B_{1}, r_{j} \in\right] s-\mu, s+\mu\left[, z_{j}\left(r_{j}\right) \in O_{\mu} y_{1}\right\}$ бесконечно.

Пусть множество $B_{3} \subseteq B_{2}$ бесконечно и для $j \in B_{3}$ отмечены точки

$$
p_{j} \in\left[s_{j}, r_{j}\right] \cap[s-\mu, s+\mu] \quad \text { и } \quad q_{j} \in\left[r_{j}, t_{j}\right] \cap[s-\mu, s+\mu]
$$

таким образом, что $z_{j}\left(\left[p_{j}, q_{j}\right]\right) \subseteq\left[O_{\mu} y_{1}\right]$.

Пусть $\varphi_{j}(t)=\left(\mathbf{e}, z_{j}(t)\right)$ для $t \in\left[p_{j}, q_{j}\right]$.

$\Phi$ ункция $\varphi_{j}$ обобщенно абсолютно непрерьвна, а в силу $(13),(4)$ и $(5)$

(14) $\varphi_{j}^{\prime}(t) \geqslant-\alpha_{j}(t)$ почти при всех $t \in\left[p_{j}, q_{j}\right]$.

Докажем, что

$(15)$ если $\varphi_{j}\left(p_{j}\right) \rightarrow C, \varphi_{j}\left(q_{j}\right) \rightarrow D$ при $j \in B_{3}$ и $j \rightarrow \infty$, то $C \leqslant D$.

Из (14) следует, что функция

$$
\psi_{j}(t)=\varphi_{j}(t)+\int_{p_{j}}^{t} \alpha_{j}(s) d s
$$

является неубывающей, поэтому $\psi_{j}\left(q_{j}\right)-\psi_{j}\left(p_{j}\right) \geqslant 0$,

$$
\left.\varphi_{j}\left(q_{j}\right)-\varphi_{j}\left(p_{j}\right) \geqslant-\int_{p_{j}}^{q_{j}} \alpha_{j}(s) d s \rightarrow 0 \quad \text { (при } j \in B_{3} \text { и } j \rightarrow \infty\right)
$$

в силу равностепенной непрерьвности первообразных функций $\alpha_{j}$. Таким образом,

$$
D-C=\lim _{j \in B_{3}, j \rightarrow \infty}\left(\varphi_{j}\left(q_{j}\right)-\varphi_{j}\left(p_{j}\right)\right) \geqslant 0,
$$

что и дает вьполнение (15).

V. Сохраняя обозначения III и IV, предположим теперь дополнительно, что

$$
\operatorname{Gr}\left(\left.z_{j}\right|_{\left[p_{j}, q_{j}\right]}\right) \rightarrow H_{1} \subseteq H \cap\left(\{t\} \times\left[O_{\mu} y_{1}\right]\right) \cap \lambda \quad \text { при } j \in B \text { и } j \rightarrow \infty
$$


и отображение $f(u)=(\mathbf{e}, u)$ инъективно на компакте $H_{1}$.

Покажем, что топология $\tau(F, s)$ и евклидова топология индуцируют на $H_{1}$ одну и ту же топологию.

Из непрерывности отображения $f$, связности и компактности множества $H_{1}$ относительно евклидовой топологии следует, что множество $f\left(H_{1}\right)$ является отрезком (или одноточечно). Обозначим его через $I$.

В силу компактности множества $H_{1}$, непрерьвности и взаимной однозначности отображения $f: H_{1} \rightarrow I$ обратное отображение $g: I \rightarrow H_{1}$ непрерьвно относительно евклидовой топологии на множестве $H_{1}$.

Для достижения поставленной цели нам достаточно проверить непрерьвность отображения $g$ относительно топологии $\tau(F, s)$.

Допустим противное. Тогда существуют такая точка $(s, a)$ множества $H_{1}$ и такое число $\varepsilon>0$, что для любого $j=1,2, \ldots$ (в силу непрерывности отображения $g$ относительно евклидовой топологии) найдется точка $a_{j} \in \lambda$, удовлетворяющая условию

$$
\left(s, a_{j}\right) \in H_{1} \cap\left(\{s\} \times\left(O_{2^{-j}} a \backslash\left(a \pm(F(s, a))_{2 \varepsilon}\right)\right)\right) .
$$

В силу компактности единичного шара мы можем перейти к подпоследовательности $\left\{\left(a_{j}-a\right) /\left\|a_{j}-a\right\|: j \in C\right\}$ последовательности $\left\{\left(a_{j}-a\right) /\left\|a_{j}-a\right\|: j=1,2, \ldots\right\}$, сходящейся к некоторому вектору $u_{0} \in \Lambda$.

Для последнего

$$
\left\|u_{0}\right\|=1 \quad \text { и } \quad u_{0} \notin \pm(F(t, a))_{2 \varepsilon} .
$$

Из условий (1) и (3) легко выводится, что

$$
(F(s, a))_{\varepsilon} \supseteq \operatorname{cc}\left(\cup\left\{F_{i}(O(s, a)): i=k, k+1, \ldots\right\}\right)
$$

для некоторого $k=1,2, \ldots$ и некоторой окрестности $O(s, a)$ точки $(s, a)$ в $U$.

В силу непрерывности отображения $g$ относительно евклидовой топологии $g([f(a)-\delta, f(a)+\delta]) \subseteq O(s, a)$ при некотором $\delta>0$.

Вектор $u_{0}$ не принадлежит ни множеству $L_{0}=\operatorname{cc}\left(\cup\left\{F_{i}(O(s, a)): i=k, k+1, \ldots\right\}\right)$, ни множеству $L_{1}=-\operatorname{cc}\left(\cup\left\{F_{i}(O(s, a)): i=k, k+1, \ldots\right\}\right)$.

По теореме Хана-Банаха существуют линейные функционалы $f_{i}: \mathbb{R}^{n} \rightarrow \mathbb{R}, i=1,2$, удовлетворяющие условию

$$
f_{i}\left(L_{i}\right) \subseteq\left[0, \infty\left[, \quad f_{i}\left((-1)^{i} u_{0}\right)<0\right.\right.
$$

В силу сходимости $a_{i} \rightarrow a$ относительно евклидовой топологии и непрерьвности отображения $f f\left(a_{i}\right) \rightarrow f(a)$ и

$$
f\left(a_{i}\right) \in[f(a)-\delta, f(a)+\delta]
$$

начиная с некоторого $i=k$.

Но тогда отрезок $M_{i}$ с конщами $f\left(a_{i}\right)$ и $f(a)$ лежит в отрезке $[f(a)-\delta, f(a)+\delta]$ и поэтому

$$
g\left(M_{i}\right) \subseteq O(s, a) .
$$


Таким образом, $\left|f\left(a_{k}\right)-f(a)\right|<\delta, a_{k} \in O(s, a), g\left(M_{i}\right) \subseteq O(s, a)$,

$$
f_{0}\left(a_{k}-a\right)<0, \quad f_{1}\left(a_{k}-a\right)>0 .
$$

Так как отображение $f$ инъективно, то $f\left(a_{k}\right) \neq f(a)$.

Рассмотрим случай, когда $f\left(a_{k}\right)>f(a)$.

По [8, теорема 4.3.10] мы можем так указать бесконечное множество $B_{4} \subseteq B_{3}$ и для $j \in B_{4}$ - точки $c_{j}, d_{j} \in\left[p_{j}, q_{j}\right]$, что $z_{j}\left(c_{j}\right) \rightarrow a, z_{j}\left(d_{j}\right) \rightarrow a_{k}$.

Кроме того, начиная с некоторого $j_{0} \in B_{4}$, график функции $z_{j}$ на отрезке с концами $c_{j}, d_{j}$ лежит в множестве $O(s, a)$ (если бы это было не так, то гомеоморфньй отрезку континуум $H_{1}$ выходил бы между точками $a$ и $a_{k}$ за пределы $O(s, a)$, что противоречит (17)).

Таким образом, в силу (15) $c_{j}<d_{j}$, начиная с некоторого $j \in B_{4}$.

Отсюда, из включения $\operatorname{Gr}\left(\left.z_{j}\right|_{\left[c_{j}, d_{j}\right]}\right) \subseteq O(s, a)$ и $(13)$ следует, что $f_{0}(a) \leqslant f_{0}\left(a_{k}\right)$, что противоречит (18).

Случай $f\left(a_{k}\right)<f(a)$ рассматривается аналогично с заменой $f_{0}$ на $f_{1}$.

VI. Вернемся к обозначениям III и положим для $j \in B_{2}$

$$
\begin{aligned}
p_{j} & =\inf \left\{p: p \in\left[s_{j}, r_{j}\right], p \geqslant s-\mu, z_{j}\left(\left[p, r_{j}\right]\right) \subseteq\left[O_{\mu} y_{1}\right]\right\} \\
q_{j} & =\sup \left\{q: q \in\left[r_{j}, t_{j}\right], q \leqslant s+\mu, z_{j}\left(\left[r_{j}, q\right]\right) \subseteq\left[O_{\mu} y_{1}\right]\right\}
\end{aligned}
$$

Тогда определенное в V множество $H_{1}$ будет неодноточечньм. Если соответствующее отображение $f$ инъективно, мы получаем требуемое из V. В противном случае при некотором $m \in f\left(H_{1}\right)$ множество $H_{2}=f^{-1}(m) \cap H_{1}$ неодноточечно. Пусть $a$ и $b-$ любые две его различные точки. В соответствии с теоремой 4.3 .10 из [8] отметим точки $p_{j}^{\prime}, q_{j}^{\prime} \in\left[p_{j}, q_{j}\right]$ таким образом, что $z_{j}\left(p_{j}^{\prime}\right) \rightarrow a, z_{j}\left(q_{j}^{\prime}\right) \rightarrow b$.

Положим для определенности (перейдя, если необходимо, к подпоследовательности), что $p_{j}^{\prime}<q_{j}^{\prime}$.

Если

(19) при любом $y \in H_{2}$ множество $F(s, y)$ содержит целиком полупространство линейного пространства $L$,

то, очевидно, на $H_{2}$ топология $\tau(F, t)$ индуцирует ту же топологию, что и евклидова топология. В противном случае возвращаемся к началу рассуждения в VI с заменой $\lambda$ на $\lambda \cap f^{-1}(m), s_{j}$ на $p_{j}^{\prime}, t_{j}$ на $q_{j}^{\prime}$ и будем делать это до тех пор, пока не добьемся вьполнения условия (19). Так как при каждом шаге размерность соответствующей плоскости $\lambda$ уменьшается на единицу, то менее чем за $n$ шагов процесс закончится. При этом мы не можем спуститься до размерности нуль, так как в этом случае нетривиальньй континуум оказался бы подмножеством плоскости размерности нуль. Теорема доказана. 


\section{СПИСОК ЦИТИРОВАННОЙ ЛИТЕРАТУРЫ}

[1] Филиппов В.В. Аксиоматическая теория пространств решений обыкновенных дифференциальных уравнений // Докл. АН СССР. 1985. Т. 280. № 2. С. 304-308.

[2] Филиппов В. В. К теории пространств решений обыкновенных дифференциальных уравнений // Докл. АН СССР. 1985. Т. 285. № 5. С. 1073-1077.

[3] Филиппов В. В. Об обыкновенных дифференциальных уравнениях с особенностями в правой части // Матем. заметки. 1985. Т. 38. №6. С. 832-851.

[4] Филиппов В.В.Два замечания к теории пространств решений обыкновенных дифференциальных уравнений // Вестн. МГУ. Сер. 1. Матем., мех. 1988. № 4. С. 53-55.

[5] Филиппов В.В. Об асимптотическом интегрировании обыкновенных дифференциальных уравнений с разрывами в правой части // Докл. АН СССР. 1991. Т. 321. №3. С. 482-485.

[6] Филиппов В.В. О стационарных точках и некоторых геометрических свойствах решений обыкновенных дифференциальных уравнений // Докл. АН СССР. 1992. Т. 323. №6. C. $1043-1047$.

[7] Филиппов В.В. Топологическое строение пространств решений обыкновенных дифференциальных уравнений // УМН. 1993. Т. 48. №1. С. 103-154.

[8] Филиппов В. В., Федорчук В. В. Общая топология. Основные конструкции. М.: Изд-во МГУ, 1988.

[9] Филиппов В.В.Пространства решений обькновенных дифференциальных уравнений. М.: Изд-во МГУ, 1994. 